\title{
Clinical Pharmacology of Elagolix: An Oral Gonadotropin-Releasing Hormone Receptor Antagonist for Endometriosis
}

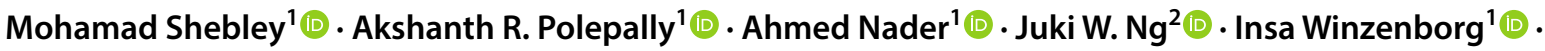 \\ Cheri E. Klein ${ }^{1}$ (1) Peter Noertersheuser ${ }^{1} \cdot$ Megan A. Gibbs $^{1} \cdot$ Nael M. Mostafa ${ }^{1}$
}

Published online: 21 November 2019

(c) The Author(s) 2019

\begin{abstract}
The clinical pharmacology of elagolix was extensively evaluated in clinical studies in healthy subjects and in women with endometriosis. Elagolix pharmacokinetics (PK) show significant population variability, however they are minimally affected by patients' baseline characteristics and demographics, except for clinically relevant extrinsic and intrinsic factors such as coadministrated strong organic anion transporting polypeptide (OATP) 1B1 inhibitors and severe hepatic impairment, which are contraindications for the use of elagolix. These studies enabled a comprehensive understanding of elagolix mechanism of action and the downstream pharmacodynamic (PD) effects on gonadotropin and ovarian hormones, as well as full characterization of the PK/PD (PKPD) relationships of elagolix at various dosages, including the approved $150 \mathrm{mg}$ once daily and $200 \mathrm{mg}$ twice daily dosing regimens for the management of moderate to severe pain associated with endometriosis. Several model-based analyses have contributed to understanding of the benefit-risk profile of elagolix in patients with endometriosis, through characterization of the exposure relationship with responder rates, with changes in bone mineral density over time, as well as the interaction with coadministered drugs. Collectively, these studies and analyses served as supportive evidence for the effectiveness of the approved dosages and provided general dosing instructions of the first approved oral gonadotropin-releasing hormone receptor antagonist.
\end{abstract}

\section{Key Points}

Elagolix is the first approved oral gonadotropin-releasing hormone $(\mathrm{GnRH})$ receptor antagonist for moderate to severe pain associated with endometriosis.

The clinical pharmacology profile of elagolix was fully characterized in several Phase 1 PKPD studies along with several model informed drug development approaches.

This comprehensive description of the clinical pharmacology attributes of elagolix provides a reference for prescribers and clinical pharmacologists who seek to use or understand the clinical PKPD properties of elagolix.

Electronic supplementary material The online version of this article (https://doi.org/10.1007/s40262-019-00840-7) contains supplementary material, which is available to authorized users.

Mohamad Shebley

mohamad.shebley@abbvie.com

1 Clinical Pharmacology and Pharmacometrics, AbbVie Inc., 1 North Waukegan Road, North Chicago, IL 60064, USA

2 Pharmaceutical Development, General Medicine, AbbVie Inc., North Chicago, IL, USA

\section{Introduction}

Elagolix (Orilissa ${ }^{\mathrm{TM}}$ ) is a novel, non-peptide oral, short-acting competitive gonadotropin-releasing hormone (GnRH) receptor antagonist approved by the US FDA for the management of moderate to severe pain associated with endometriosis [1], and is currently in development for the management of heavy menstrual bleeding associated with uterine fibroids [2, 3]. Both endometriosis and uterine fibroids are estrogen-dependent conditions [2, 4], and elagolix suppresses gonadotropin hormones and ovarian estrogen production in a dose-dependent manner, modulating circulating estrogen levels from partial suppression of estradiol (E2) at lower doses to nearly full suppression at higher doses [5, 6]. This is in contrast to GnRH receptor agonists, which, after an initial stimulatory phase (flare effect), desensitize the pituitary GnRH receptors and lead to profound suppression of ovarian sex steroid secretion similar to that of ovariectomized women [6].

The clinical development program for elagolix included several clinical pharmacology studies, which enabled full characterization of the pharmacokinetics (PK), pharmacodynamics (PD), effects of intrinsic and extrinsic factors, and population $\mathrm{PK} / \mathrm{PD}$, exposure-response (safety and efficacy) 
analyses and physiologically based PK (PBPK) modeling and simulation. A summary of the extensive data, analyses, and conclusions generated from these studies is presented herein to offer a comprehensive overview of the clinical pharmacology attributes of elagolix.

\section{Mechanism of Action of Elagolix}

Elagolix is a highly potent $\left(K_{\mathrm{D}}=54 \mathrm{pM}\right) \mathrm{GnRH}$ receptor antagonist that inhibits endogenous $\mathrm{GnRH}$ signaling by binding competitively to GnRH receptors in the anterior pituitary gland [7]. Elagolix mechanism of action (MoA) is different from long-acting GnRH receptor agonists, which induce 1-2 weeks of 'flare-up' by downregulating GnRH receptors [6]. The competitive nature of elagolix competitive antagonism of the GnRH receptors provides an advantage by allowing for rapid and reversible onset and offset, and hence more flexibility in modulating the hypothalamic-pituitary-gonadal axis. An illustration that describes elagolix
MoA and downstream effects on gonadotropins and ovarian hormones is shown in Fig. 1.

\section{Pharmacokinetics (PK) of Elagolix}

\subsection{Absorption}

Elagolix sodium is a non-peptide, orally bioavailable small molecule, amorphous solid that is freely soluble in water. At either the 150 or $200 \mathrm{mg}$ dose, elagolix is highly soluble per the Biopharmaceutics Classification System (BCS) throughout the physiological $\mathrm{pH}$ range, it exhibits high aqueous solubility (approximately $1 \mathrm{mg} / \mathrm{mL}$ ), is a zwitterion with $\mathrm{p} K_{\mathrm{a}} 4.0$ (acid) and 7.9 (base), and has an apparent low to moderate permeability $\left(0.5-2.8 \times 10^{-6} \mathrm{~cm} / \mathrm{s}\right)$ based on in vitro Caco-2 studies [7]. These data suggest that elagolix could be classified as a BCS class III drug. Elagolix contains one chiral center and is manufactured exclusively as the $(R)$-isomer. The structural formula of elagolix sodium is shown in Fig. 2.

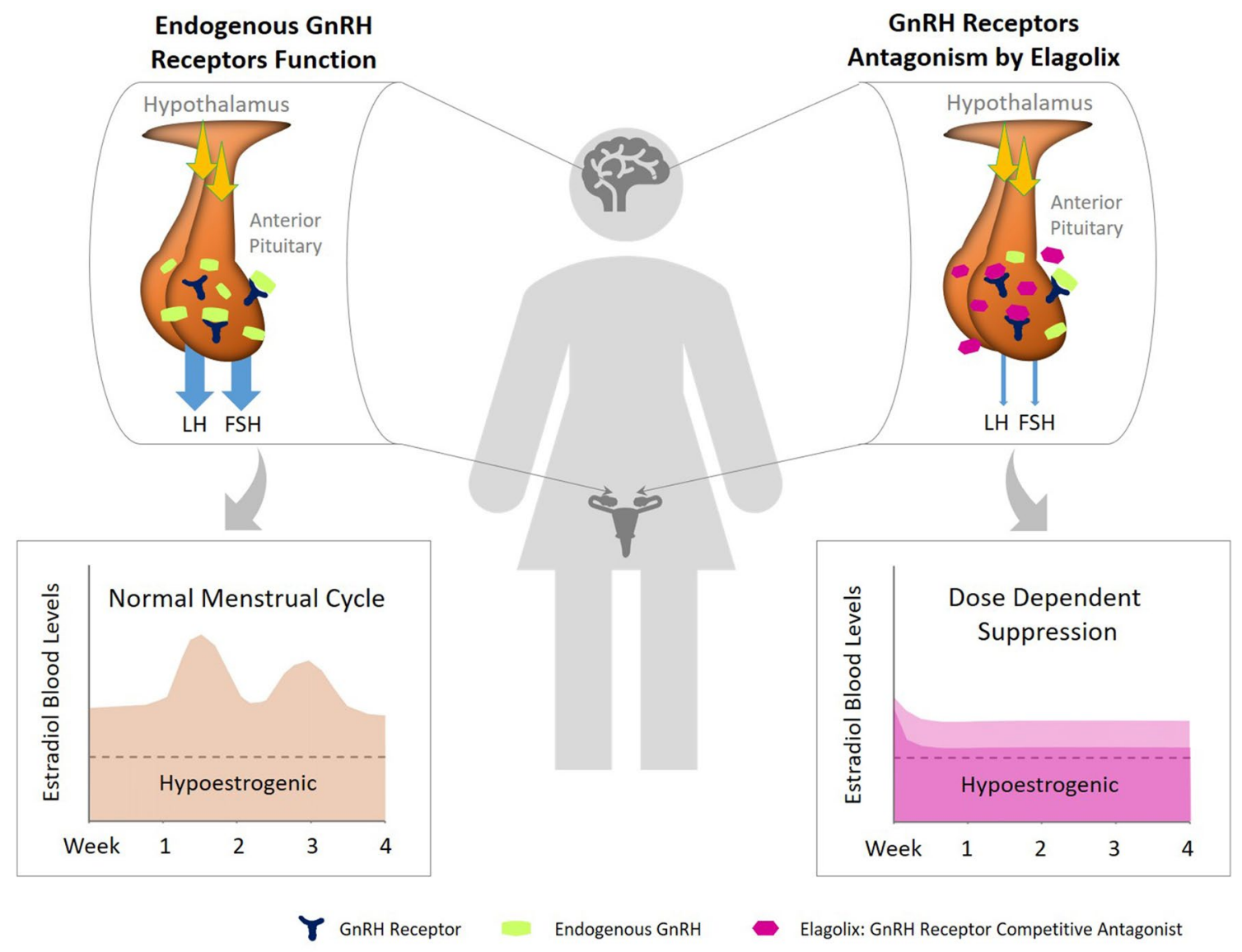

Fig. 1 Illustration of GnRH action and function during the normal female menstrual cycle, elagolix mechanism of GnRH receptors' competitive antagonism at the anterior pituitary gland, and its down- stream dose-dependent effects on circulating estradiol levels in blood. $\mathrm{GnRH}$ gonadotropin-releasing hormone 
<smiles>COc1cccc(-c2c(C)n(Cc3c(F)cccc3C(F)(F)F)c(=O)n(C[C@@H](NCCCC(=O)O[NH3+])c3ccccc3)c2=O)c1F</smiles>

Fig. 2 Structure of elagolix sodium; molecular weight of 653.58 . Elagolix free acid has a molecular weight of 631.60

In clinical PK studies in healthy subjects, elagolix absorption is rapid, with a time to maximum concentration $\left(T_{\max }\right)$ of approximately $1 \mathrm{~h}$. Elagolix exposure (maximum concentration $\left[C_{\max }\right]$ and area under the curve [AUC]) is dose proportional from 100 to $400 \mathrm{mg}$ twice daily [5], and more than dose proportional with single doses of 600-1200 mg. A regional absorption study was conducted in six healthy subjects to characterize the PK of elagolix $100 \mathrm{mg}$ administered to the stomach via oral solution, and to the jejunum, ileum, and colon via a radiolabeled remote drug delivery capsule (InteliSite ${ }^{\circledR}$ ). Based on the geometric mean AUC values, elagolix doses delivered as either a solution to the stomach or in an InteliSite ${ }^{\circledR}$ capsule to the jejunum and ileum resulted in comparable overall systemic exposure, with a geomean
AUC from time zero to infinity $\left(\mathrm{AUC}_{\infty}\right)$ of $432.7,411.8$, and $443.9 \mathrm{ng} \bullet \mathrm{h} / \mathrm{mL}$, respectively. However, the geomean $\mathrm{AUC}_{\infty}$ for the colon administration was only $35.22 \mathrm{ng} \bullet \mathrm{h} / \mathrm{mL}$, representing $<10 \%$ that of the same dose deposited into the stomach/duodenum, jejunum, or ileum, and with a mean $C_{\max }$ for the colon administration at approximately 38 times lower than that following administration into the ileum (Electronic Supplementary Fig. 1).

Several elagolix formulations ranging from suspension to modified and immediate-release (IR) tablets were evaluated throughout the development program and across multiple phase I studies. While variability in the PK profiles of elagolix was observed across the tested formulations, the exposures did not vary significantly, consistent with a characteristic BCS III behavior. An IR tablet formulation was chosen for the endometriosis phase III studies, as well as for the commercial formulation. The final commercial tablet formulation of elagolix is bioequivalent to the phase III tablet formulation. Representative PK profiles of the phase III and commercial $200 \mathrm{mg}$ IR tablet formulations is shown in Fig. 3. Elagolix $150 \mathrm{mg}$ once-daily and $200 \mathrm{mg}$ twice-daily PK parameters at steady state are summarized in Table 1 . Dose proportional PK are demonstrated for both elagolix dosages based on the dose normalized $C_{\max }$ and AUC values; elagolix does not accumulate with repeated once daily or twice daily dosing.

\subsection{Food Effect}

The effect of food on elagolix plasma exposure was assessed following administration of a high-fat meal in a pivotal phase
Fig. 3 Elagolix plasma concentration-time profiles of the phase III and commercial formulations of Orilissa. Symbol represents the mean

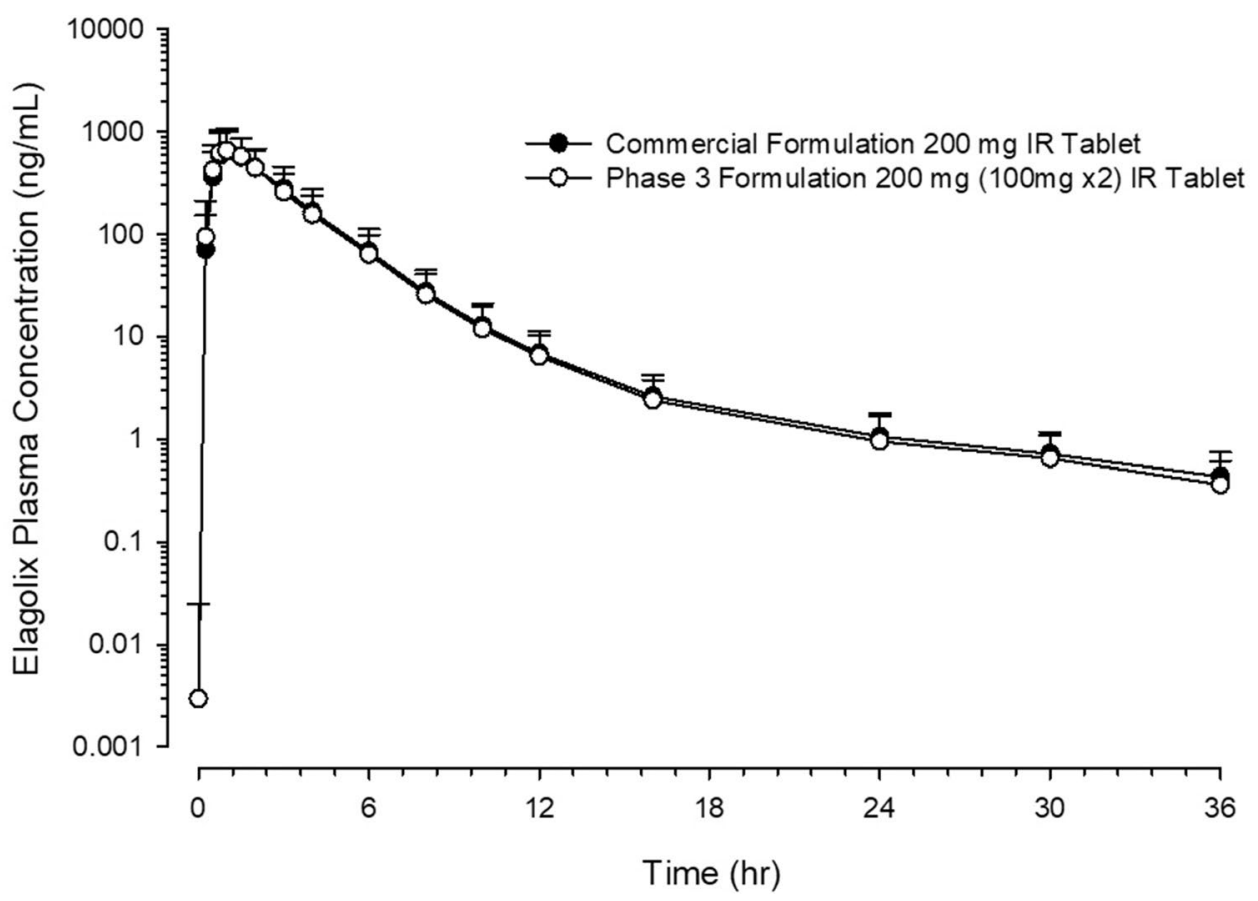


Table 1 Mean (percentage coefficient of variation) pharmacokinetic parameters at steady state of elagolix $150 \mathrm{mg}$ qd or $200 \mathrm{mg}$ bid

\begin{tabular}{lll}
\hline Pharmacokinetic parameters & $150 \mathrm{mg} \mathrm{qd}$ & $200 \mathrm{mg}$ bid \\
\hline$T_{\max }\left(\mathrm{h}^{\mathrm{a}}\right)$ & $1.0(0.5-1.0)$ & $1.0(0.5-1.5)$ \\
$C_{\max }(\mathrm{ng} / \mathrm{mL})$ & $574(29)$ & $774(68)$ \\
$\mathrm{AUC}_{\tau}(\mathrm{ng} \times \mathrm{h} / \mathrm{mL})$ & $1292(31)$ & $1725(57)$ \\
$t_{1 / 2}\left(\mathrm{~h}^{\mathrm{b}}\right)$ & $6.42 \pm 3.20$ & $4.29 \pm 0.47$ \\
$\mathrm{CL} / F(\mathrm{~L} / \mathrm{h})$ & $123(21)$ & $144(43)$ \\
$V_{\mathrm{ss}} / F(\mathrm{~L})$ & $1674(94)$ & $881(38)$ \\
$R_{\mathrm{ac}}$ & $0.98(7)$ & $0.89(19)$ \\
$C_{\mathrm{max}} / \mathrm{dose}$ & $3.83(29)$ & $3.87(68)$ \\
$\mathrm{AUC}_{\tau} / \mathrm{dose}$ & $8.61(31)$ & $8.62(57)$ \\
\hline
\end{tabular}

$A U C_{\tau}$ area under the concentration-time curve during the dosing interval $(\tau)$ of $24 \mathrm{~h}$ for once-daily administration and $12 \mathrm{~h}$ for twicedaily administration, bid twice daily, $C_{\max }$ maximum concentration, $C L / F$ apparent clearance, $q d$ once daily, $R_{a c}$ accumulation ratio, $T_{\max }$ time to maximum concentration, $t_{1 / 2}$ terminal elimination half-life, $V_{s s} / F$ apparent volume of distribution at steady state

${ }^{a}$ Data are reported as median (range)

${ }^{b}$ Data are reported as harmonic mean \pm pseudo-standard deviation

I bioavailability study. With the high-fat meal condition, a slight reduction in elagolix plasma exposure relative to the fasted condition was observed, with a decrease of $24 \%$ and $36 \%$ in $\mathrm{AUC}_{\infty}$ and $C_{\max }$, respectively (Fig. 4). In three of four endometriosis phase III pivotal trials, subjects were instructed to administer elagolix at least $1 \mathrm{~h}$ before or $2 \mathrm{~h}$ after a meal to avoid a potential lower exposure of elagolix. However, since administration of a high-fat meal demonstrated the worst-case scenario for the effect of food on elagolix exposure, and due to the lack of clinical significance of the small reduction in elagolix exposure with meals, elagolix was administered without regard to meals in the fourth endometriosis phase III extension trial. Elagolix clinical efficacy results from all the phase III studies were similar, regardless of drug administration instructions with respect to meals; therefore, Orilissa is administered with or without food [1].

\subsection{Distribution}

Elagolix is moderately ( $80 \%$ ) bound to human plasma proteins, and preferentially partitions into plasma rather than blood cellular components, with blood-to-plasma ratios of 0.6 [1]. Based on population PK analyses using data from five phase I healthy volunteers and four phase III endometriosis patient studies, the elagolix estimated apparent central volume of distribution $\left(V_{\mathrm{c}} / F\right)$ was $257 \mathrm{~L}$ [8]. Elagolix is a substrate of the hepatic uptake transporter organic anion transporting polypeptide (OATP) 1B1 based on in vitro studies [10], pharmacogenetics analysis of OATP1B1 variants [8], and clinical drug-drug interactions (DDIs) with singledose rifampin [16]; however, population PK analysis did not identify the OATP1B1 genotype as a significant covariate on elagolix $V_{\mathrm{c}} / F[8]$.

\subsection{Metabolism}

Elagolix is metabolized by multiple cytochrome P450 (CYP) enzymes in vitro, with predominant contribution from
Fig. 4 Elagolix $200 \mathrm{mg}$ commercial IR tablet formulation plasma concentration-time profiles under fasted and fed (high-fat meal) conditions in healthy subjects. Symbol represents the mean, error bars are the standard deviation. $I R$ immediate-release

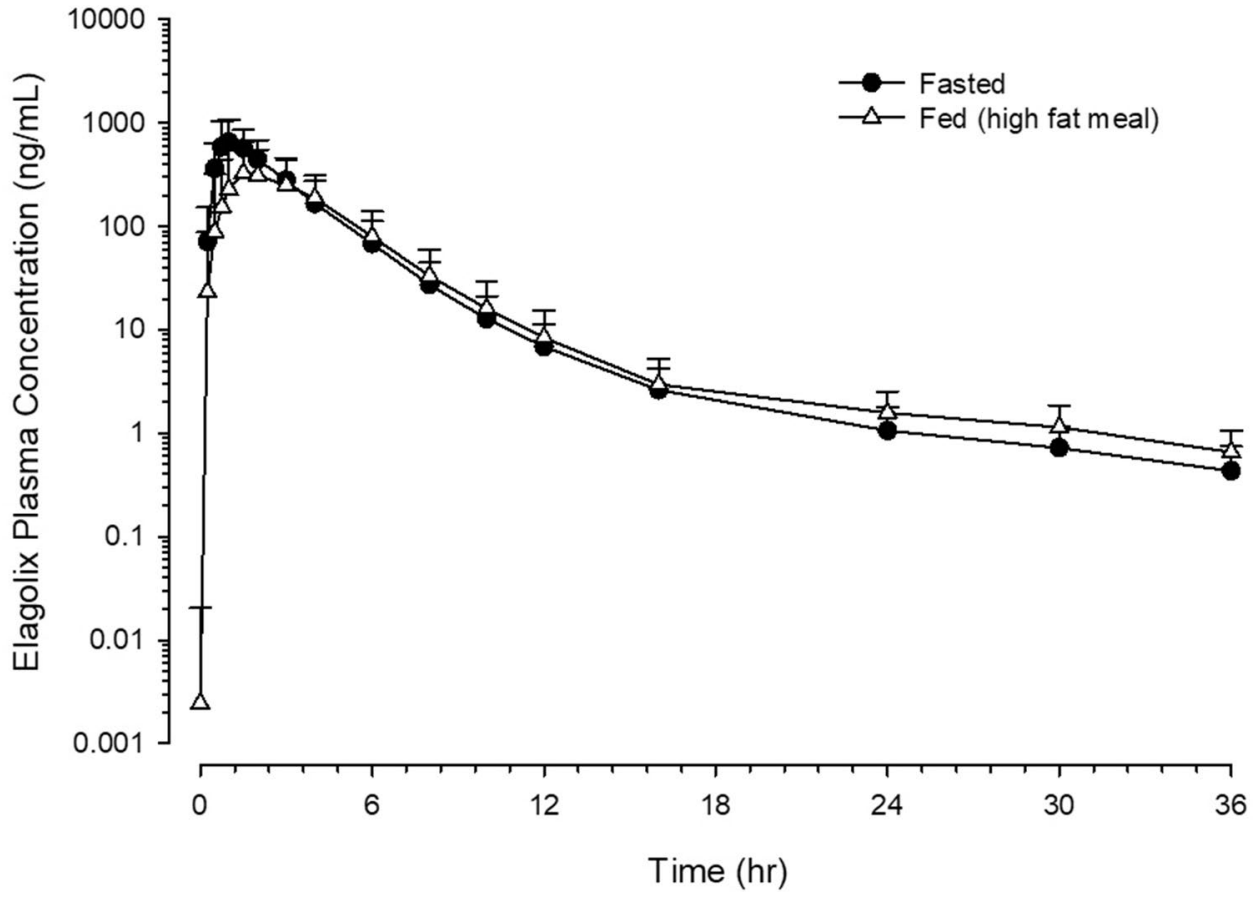


CYP3A4 (approximately 50\%) [9], and minor contributions from other CYPs. In a mass balance study in humans, following administration of a single oral dose of $150 \mathrm{mg}$ of $\left[{ }^{14} \mathrm{C}\right]$ elagolix to six healthy subjects, elagolix was the predominant form of radioactivity in plasma. Mean AUC $\infty$ values for the $O$-demethyl (M1) and $N$-dealkylated (M2) metabolites were approximately $2.4 \%$ and $3.3 \%$, respectively, of the mean elagolix exposure (Fig. 5). Of the administered dose, $69 \%$ was recovered in feces and urine as metabolites, and a total of 11 minor metabolites were identified in plasma, each representing $<3 \%$ of total plasma radioactivity. None of the metabolites in human plasma were classified as major or disproportionate metabolites. In addition to CYPmediated metabolites, a minor trace of an acyl-glucuronide metabolite in urine was detected, suggesting a minor contribution from uridine 5 '-diphospho-glucuronosyltransferase (UGT) enzymes. Metabolite profiling of the feces (primary route of elimination, indicating biliary excretion) revealed that approximately $38 \%$ of the radioactive dose was eliminated as the M1 metabolite, 26\% was unchanged elagolix, with the remainder being a combination of multiple minor metabolites. These data suggest that unchanged elagolix is the major drug-derived material in human plasma and elagolix is extensively metabolized.

\subsection{Elimination}

The elagolix concentration-time profile exhibits a biphasic characteristic after reaching $C_{\max }$, with an apparent terminal elimination half-life $\left(t_{1 / 2}\right)$ of approximately $4-6 \mathrm{~h}$ in healthy subjects $[5,10]$. Thus, repeated dosing of elagolix once or twice daily does not result in significant drug accumulation in plasma.

In the mass balance single-dose study, $90.1 \%$ of total radioactivity was excreted in the feces, and urinary excretion accounted for only $2.9 \%$ of total radioactivity, with mean total recovery of $93 \%$ by approximately 9 days after dosing.

The minor urinary excretion of radioactivity was consistent with the population PK analysis, where renal function was not associated with elagolix PK parameters [8]. The OATP1B1 genotype was a statistically significant covariate on apparent clearance $(\mathrm{CL} / \mathrm{F})$, however the small change in $\mathrm{CL} / \mathrm{F}(14 \%)$ was not considered clinically relevant when the poor and intermediate transporter genotypes were combined and compared with the extensive transporter genotype [8]. Figure 6 depicts the disposition and elimination mechanisms of elagolix in humans.

\section{Pharmacodynamics of Elagolix}

\subsection{Effects on Hormones, Folliculogenesis, and Ovulation}

Administration of elagolix results in dose-dependent suppression of the gonadotropins, luteinizing hormone (LH) and follicle-stimulating hormone (FSH), leading to decreased blood concentrations of the ovarian sex hormones E2 and progesterone $(\mathrm{P})$. In a multiple-ascending dose study in premenopausal healthy female subjects, elagolix $150 \mathrm{mg}$
Fig. 5 Elagolix mean plasma concentration-time profiles and total plasma radioactivity (elagolix and metabolites M1 and M2)

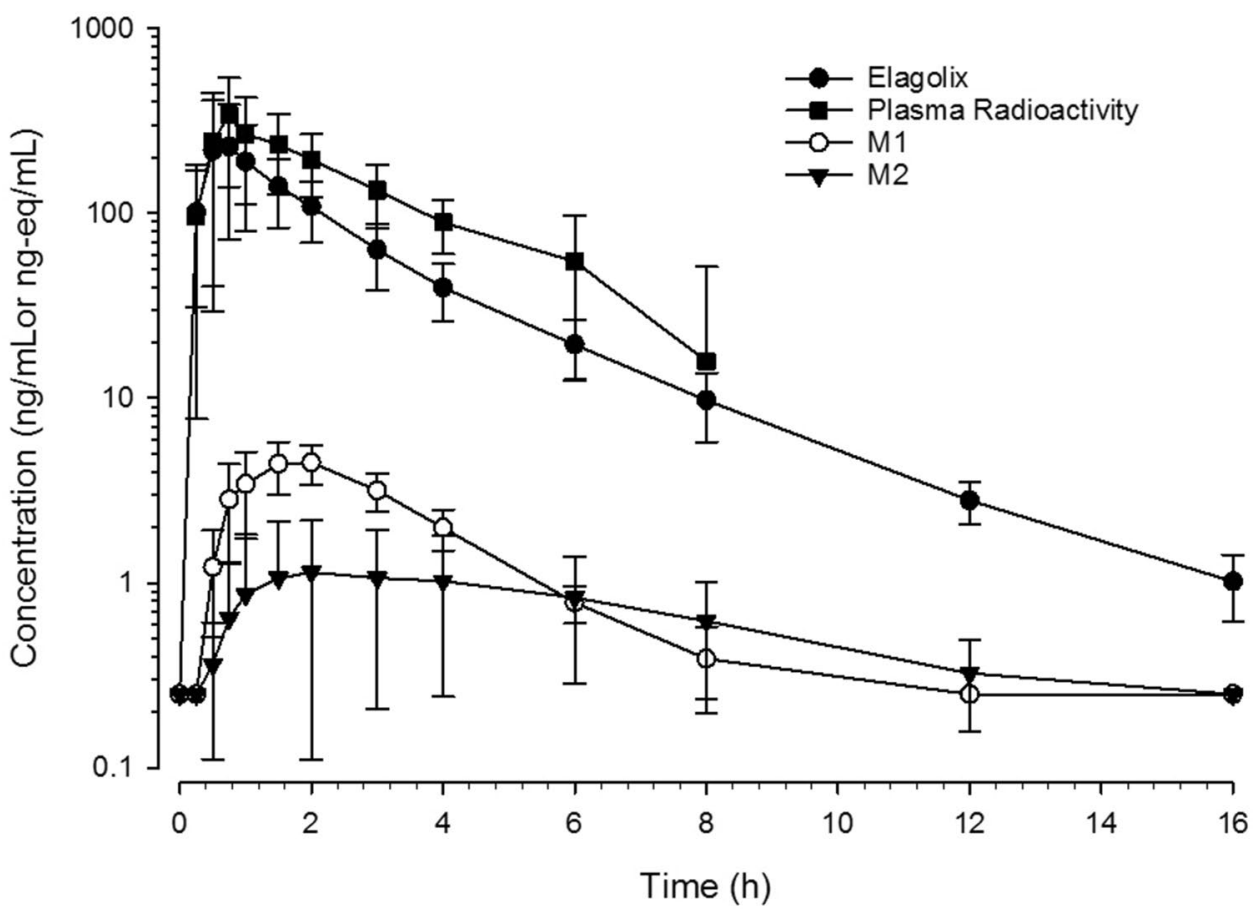


Fig. 6 Elagolix mechanisms of disposition via hepatic uptake transporter OATP1B1, metabolism by CYP3A and other CYP and UGT enzymes, and biliary elimination. OATP organic anion transporting polypeptide, CYP cytochrome $\mathrm{P} 450$, UGT uridine 5'-diphosphoglucuronosyltransferase

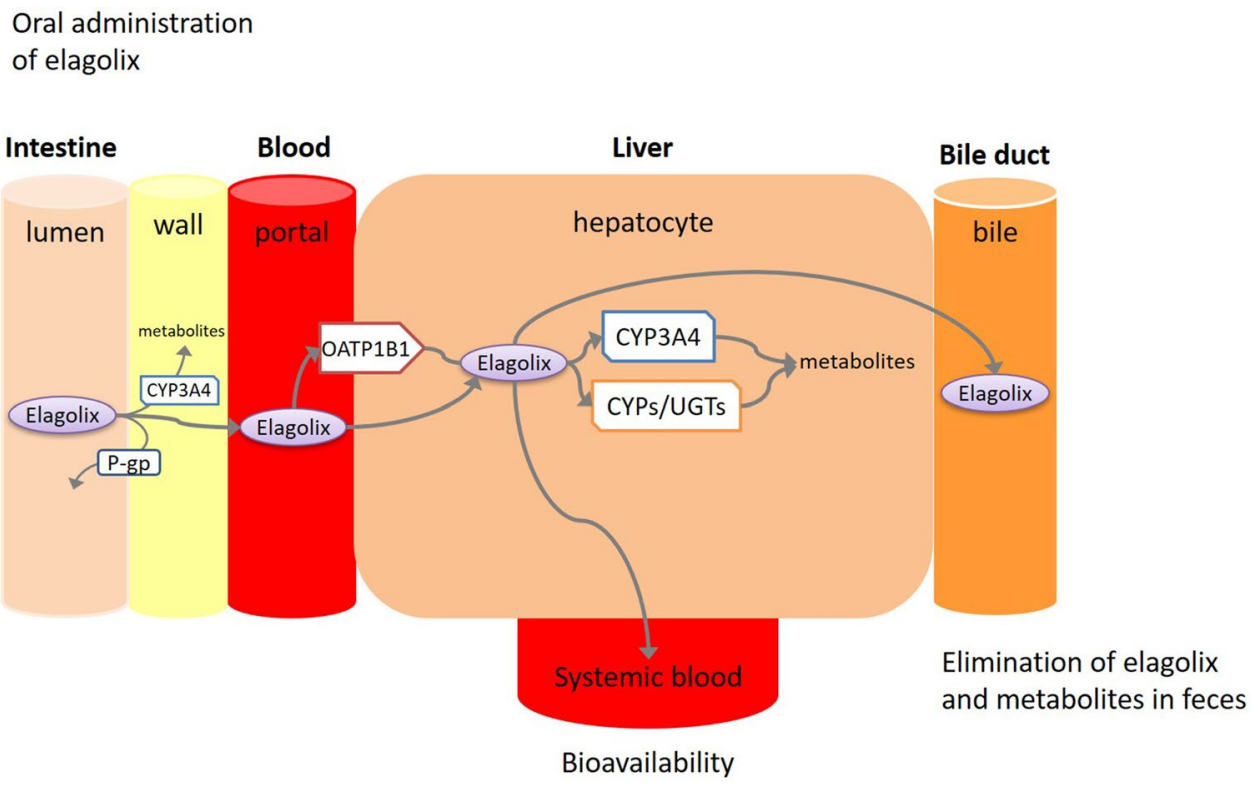

once daily, or 100, 200, 300, or $400 \mathrm{mg}$ twice daily, or placebo, was administered for 21 days [5]. Dose-dependent suppression of sex hormones was achieved rapidly within hours after administration of the first dose on day 1 and continued through day 21, with maximum E2 suppression achieved with elagolix doses of $200 \mathrm{mg}$ twice daily or higher. At elagolix doses $\geq 100 \mathrm{mg}$ twice daily, P concentrations remained at anovulatory levels throughout 21 days of dosing. Dose-dependent suppression of FSH and LH was also observed, with maximal or near-maximal suppression achieved at elagolix doses of $300 \mathrm{mg}$ twice daily and $200 \mathrm{mg}$ twice daily, respectively. LH and FSH were suppressed compared with placebo, however LH suppression was more pronounced than that of FSH in all groups except the $150 \mathrm{mg}$ once daily group. When elagolix administration was stopped, LH and FSH levels rose within $24 \mathrm{~h}$ after the last dose, and E2 levels began to rise $24 \mathrm{~h}$ after the last dose was administered [5]. The effects of different doses and dosing regimens of elagolix alone or with the hormonal add-back therapy standard dose Activella ${ }^{\circledR}$ (E2/norethindrone acetate, $1 / 0.5 \mathrm{mg}$ ) on ovulation, ovarian activity, and ovarian reserve were evaluated in an open-label study in healthy adult premenopausal females [11]. During the 3-month treatment phase, with three times weekly hormone sampling, suppression of gonadotropins and ovarian hormones were observed in a dose-dependent manner. Mean E2 levels observed at the $150 \mathrm{mg}$ once-daily dose were approximately $40-50 \mathrm{pg} / \mathrm{mL}$, consistent with partial E2 suppression. On the other hand, and consistent with the previous study, near maximal suppression was observed with the 200 and $300 \mathrm{mg}$ twice-daily regimens, with mean E2 levels of approximately $20-40 \mathrm{pg} /$ $\mathrm{mL}$. When standard dose Activella was administered with the elagolix $300 \mathrm{mg}$ twice-daily regimen, mean E2 levels appeared to increase to slightly above the levels observed with the $150 \mathrm{mg}$ once-daily regimen due to exogenous E2 administration. Comparing the E2 levels among the low dose Activella and other add-back therapies [28], the standard dose of Activella provided optimal levels of E2 when administered exogenously with elagolix. In the endometriosis pivotal phase III trials, the serum E2 profiles in endometriosis patients were similar to those in healthy subjects. The monthly average E2 concentrations in endometriosis patients demonstrated dose-dependent suppression with the $150 \mathrm{mg}$ once-daily and $200 \mathrm{mg}$ twice-daily regimens. The average E2 levels across placebo or elagolix treatments were maintained throughout the 6 months of the treatment period, with $150 \mathrm{mg}$ once daily demonstrating partial suppression, and $200 \mathrm{mg}$ twice daily demonstrating nearly full suppression, relative to placebo (Fig. 7).

Elagolix dose-dependently suppressed ovulation and ovarian activity and decreased endometrial thickness when compared with screening values. Across elagolix dosages ranging from $100 \mathrm{mg}$ once daily to $300 \mathrm{mg}$ twice daily, there was no trend of increasing endometrial thickness. Elagolix did not affect anti-mullerian hormone levels or ovarian reserve at any dose level. Although elagolix was able to suppresses gonadotrophic hormones and ovulation, it is not considered a contraceptive.

\subsection{QT Interval}

A placebo- and active-controlled (moxifloxacin $400 \mathrm{mg}$ ), randomized, single-dose (300 or $1200 \mathrm{mg}$ ), four-period, four-sequence crossover study was conducted to evaluate the potential for QTc interval prolongation by elagolix in healthy premenopausal adult females. From elagolix doses of 
Fig. 7 Monthly average estradiol levels in endometriosis patients during the pivotal phase III trials

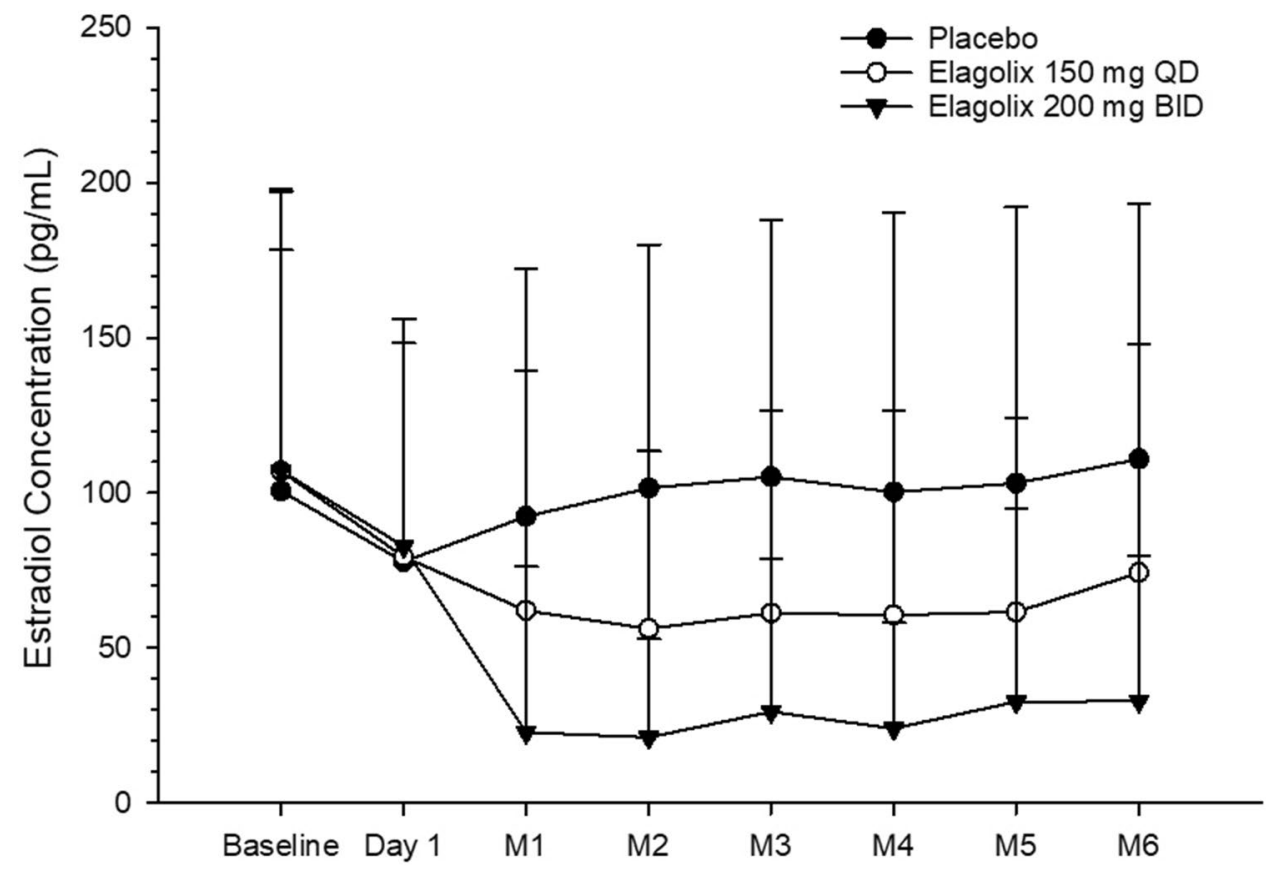

300-1200 mg, the $C_{\max }$ appeared to increase approximately 10-fold, and the $\mathrm{AUC}_{\mathrm{t}}$ increased approximately 14-fold. Elagolix peak concentrations in subjects administered a single dose of $1200 \mathrm{mg}$ was 17-fold higher than the concentration in subjects administered elagolix $200 \mathrm{mg}$ twice daily. For both doses, the baseline-adjusted QT interval corrected for heart rate using Fridericia's correction formula (QTcF), compared with placebo $(\Delta \Delta \mathrm{QTcF})$, was $<10 \mathrm{~ms}$ at all the time points evaluated. Elagolix does not cause clinically relevant prolongation of the QTc interval [12].

\section{Intrinsic Factors}

\subsection{Race/Ethnicity}

Elagolix PK and resulting hormone profiles were evaluated in Japanese and Han Chinese subjects after multiple doses of $150 \mathrm{mg}$ once daily or $200 \mathrm{mg}$ twice daily. Elagolix AUC from Japanese and Han Chinese subjects were comparable (approximately 7-20\% higher) with exposures from Western subjects [13]. With no clinically meaningful PK or PD differences, elagolix dose adjustment is not warranted for Asian subjects.

\subsection{Renal Function}

The PK of elagolix in women with moderate to severe renal impairment (estimated glomerular filtration rate [eGFR] $\geq 15$ and $<60 \mathrm{~mL} / \mathrm{min} / 1.73 \mathrm{~m}^{2}$ ) and end-stage renal disease (ESRD, including women receiving dialysis)
[eGFR $<15 \mathrm{~mL} / \mathrm{min} / 1.73 \mathrm{~m}^{2}$ ] were evaluated following a single dose of elagolix $200 \mathrm{mg}$. The mean elagolix $C_{\max }$ and AUC were comparable between women with normal renal function and those with ESRD. In women with moderate to severe renal impairment, the overall exposure was approximately $26 \%$ lower than that observed in women with normal renal function [14]. Elagolix $T_{\max }$ and $t_{1 / 2}$ were comparable among subjects with normal renal function and those with renal impairment. The unbound fractions of elagolix were also similar among subjects with normal renal function, moderate to severe renal impairment, and ESRD. Elagolix dose adjustment is not required in women with any degree of renal impairment or ESRD (including women receiving dialysis).

\subsection{Hepatic Function}

The PK of elagolix in women with mild (Child-Pugh A), moderate (Child-Pugh B), or severe (Child-Pugh C) hepatic impairment were evaluated following a single dose of elagolix $150 \mathrm{mg}$ [14]. In women with mild hepatic impairment (Child-Pugh A), elagolix exposures are comparable $(<25 \%$ difference) with women with normal hepatic function; thus, dose adjustment is not required in women with mild hepatic impairment (Child-Pugh A). In women with moderate hepatic impairment (Child-Pugh B), elagolix $C_{\max }$ and AUC were increased by $160 \%$ and $170 \%$, respectively. In these women, the $150 \mathrm{mg}$ once-daily dose is recommended, with a maximum of 6 months' treatment duration, because elagolix exposure would be comparable with that of the $200 \mathrm{mg}$ twice-daily dose in women with normal hepatic function. 
The $200 \mathrm{mg}$ twice-daily dosing regimen is not recommended in women with moderate hepatic impairment (Child-Pugh B) $[1]$.

In women with severe hepatic impairment (Child-Pugh C), elagolix $C_{\max }$ and AUC were increased by $520 \%$ and $570 \%$, respectively [14]. Hence, elagolix is contraindicated in women with severe hepatic impairment (Child-Pugh C) [1].

\subsection{Pharmacogenetics}

Pharmacogenetic analysis was conducted to assess the impact of variants in the OATP1B1/SLCO1B1 gene on subject's exposure to elagolix. The SLCO1B1 genetic variant $521 \mathrm{~T}>\mathrm{C}$ genotype was assayed to classify subjects into one of three OATP1B1 transporter genotype statuses, i.e. extensive transporter (ET, homozygous wild-type 521T $>\mathrm{C}$ ), intermediate transporter (IT, heterozygous for $521 \mathrm{~T}>\mathrm{C}$ ), and poor transporter (PT, homozygous variant $521 \mathrm{~T}>\mathrm{C}$ ). A total of 1314 DNA samples from four phase III studies in subjects with moderate to severe endometriosis-associated pain, and a total of 462 samples from 19 phase I studies, were genotyped. The results suggest that $72 \%$ of subjects were ET, $25 \%$ were IT, and $2.5 \%$ were PT. The disposition of elagolix involves OATP1B1, and higher (less than twofold) plasma concentrations of elagolix were observed in groups of patients and healthy subjects who have two reduced function alleles of the gene that encodes OATP1B 1 (SLCO1B1 521T > C). The frequency of this SLCO1B1 521 $\mathrm{C} / \mathrm{C}$ genotype is generally $<5 \%$ in most racial/ethnic groups [8]. The lack of clinical relevance of OATP1B1 genotypes on elagolix exposure is discussed in a later section on population $\mathrm{PK}$ analysis.

\section{Extrinsic Factors}

\subsection{Mechanism-Based Drug-Drug Interactions (DDIs)}

Mechanism-based DDI studies evaluated the effects of coadministration of CYP3A/P-glycoprotein (P-gp) and OATP1B1/P-gp inhibitors and CYP3A inducers on the PK of elagolix (victim), as well as the effects of elagolix (perpetrator) on the PK of CYP3A, P-gp, OATP1B1 and breast cancer-resistance protein (BCRP) substrates.

\subsection{Elagolix as a Victim of Cytochrome P450 s (CYPs) and Transporter-Mediated DDIs}

Following elagolix coadministration with ketoconazole, elagolix $C_{\max }$ and AUC increased 1.77- and 2.20-fold, respectively, compared with elagolix alone, indicating that elagolix is not a sensitive substrate of CYP3A according to the FDA criteria [9, 15]. Despite these small changes in elagolix exposure, the duration of treatment is limited to 6 months with $150 \mathrm{mg}$ once daily and 1 month with $200 \mathrm{mg}$ twice daily, with concomitant administration of strong CYP3A inhibitors to avoid any potential BMD changes due to increased elagolix exposure.

Elagolix $C_{\max }$ and AUC increased 4.37- and 5.58-fold, respectively, when elagolix was coadministered with a single dose of rifampin (OATP1B1 and P-gp inhibitor), and 2.0- and 1.65-fold, respectively, after multiple doses of rifampin (CYP3A and P-gp inducer) compared with elagolix alone [16]. The increase in elagolix exposures with a single dose of rifampin may be attributed to OATP1B1 (and perhaps intestinal $\mathrm{P}-\mathrm{gp}$ ) inhibition. The increase in elagolix exposures with multiple doses of rifampin is likely due to the net effect resulting from acute OATP1B1 inhibition and CYP3A/P-gp induction. Because other strong inhibitors of OATP1B1 (i.e. cyclosporine) do not pose induction potential for CYP3A/P-gp, and the potential magnitude of increase in elagolix exposures with such inhibitors may be similar to that caused by single-dose rifampin, concomitant use of strong inhibitors of OATP1B1 is contraindicated with Orilissa. Figure 8 summarizes the clinically relevant DDI results of elagolix changes in exposure upon coadministration with CYP3A/P-gp modulators, which informed dosing instructions in the United States package insert (USPI) for Orilissa [1].

\subsection{Elagolix as a Perpetrator of CYPs and Transporter-Mediated DDIs}

In vitro data suggested that elagolix is a weak to moderate inducer of CYP3A [12]. The elagolix $150 \mathrm{mg}$ once daily and $300 \mathrm{mg}$ twice daily dosing regimens decreased midazolam (sensitive CYP3A substrate) AUC by $35 \%$ and $55 \%$, respectively. Therefore, elagolix is clinically classified as a weak to moderate CYP3A inducer [17].

Elagolix is an inhibitor of the hepatic uptake transporter OATP1B1 and efflux transporters P-gp and BCRP, in vitro [12].

Following coadministration of elagolix as single $(200 \mathrm{mg})$ or multiple (200 mg twice daily) doses with digoxin, the $C_{\max }$ and AUC of digoxin increased approximately 1.70- and 1.30 -fold, respectively [18]. Due to the narrow therapeutic window of digoxin, clinical monitoring of digoxin is recommended when coadministered with elagolix.

Coadministration of rosuvastatin $20 \mathrm{mg}$ once daily (steady-state) with the first $300 \mathrm{mg}$ dose of elagolix increased rosuvastatin $C_{\max }$ by 1.67 -fold, whereas rosuvastatin AUC from time zero to $24 \mathrm{~h}\left(\mathrm{AUC}_{24}\right)$ was not altered. This may be explained by the inhibition of OATP1B1 and/ or BCRP transporters by the single $300 \mathrm{mg}$ dose of elagolix. 


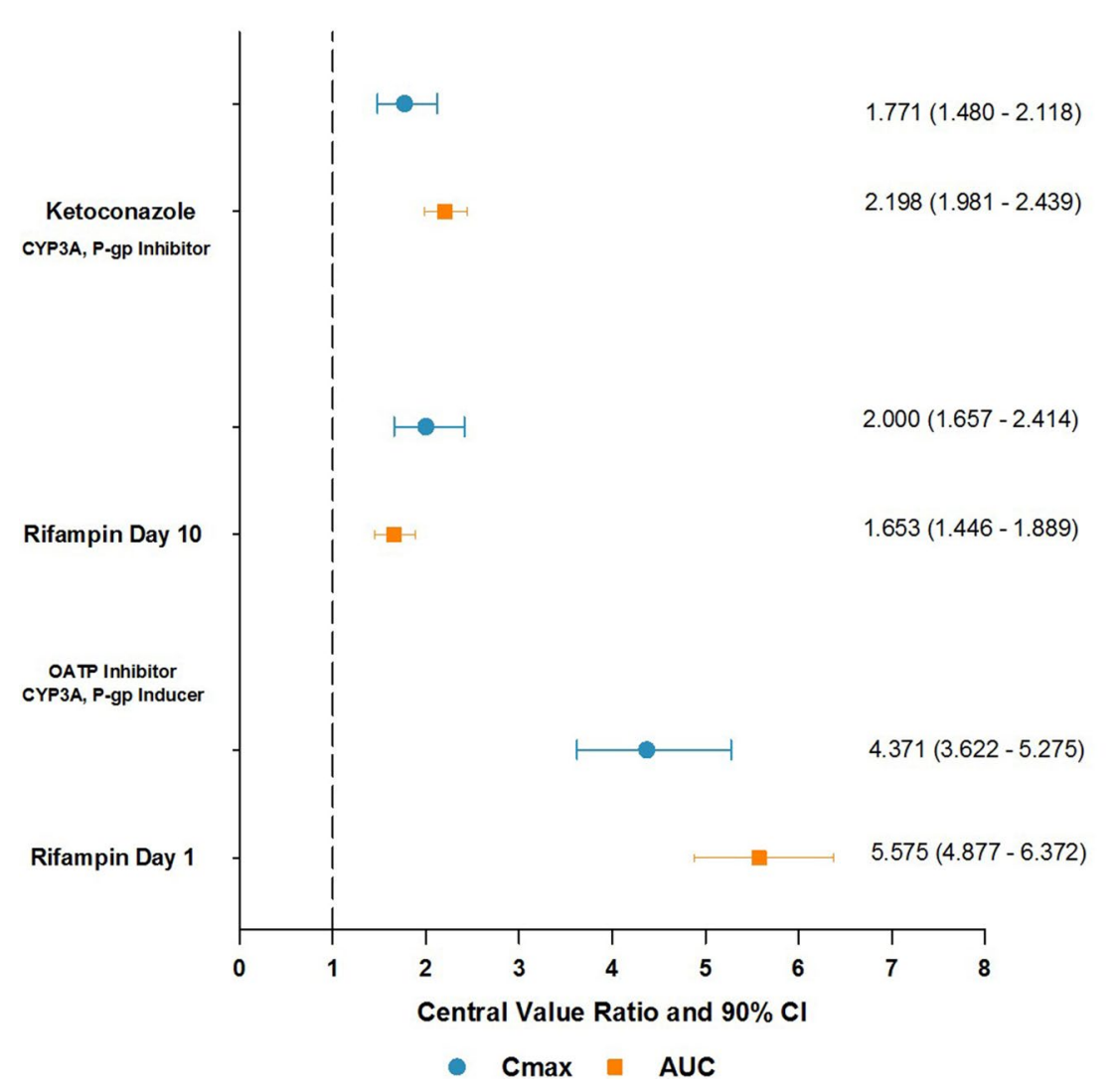

\section{Clinical Relevance}

Limit concomitant use of

Orilissa $150 \mathrm{mg}$ QD and

strong CYP3A inhibitors to 6

months. Concomitant use

of Orilissa $200 \mathrm{mg}$ BID and

strong CYP3A inhibitors for

more than 1 month is not

recommended

Limit concomitant use of

Orilissa $150 \mathrm{mg}$ QD and

rifampin to 6 months.

Concomitant use of Orilissa

$200 \mathrm{mg} \mathrm{BID}$ and rifampin is

not recommended

Fig. 8 Effect of coadministered drugs on elagolix exposures, and clinical implications for dosing

Coadministration of rosuvastatin $20 \mathrm{mg}$ once daily (steady state) with elagolix $300 \mathrm{mg}$ twice daily resulted in comparable rosuvastatin $C_{\text {max }}$, and decreased rosuvastatin $\mathrm{AUC}_{24}$ by $40 \%$. The mechanism(s) for the decrease in rosuvastatin AUC when coadministered with multiple-dose elagolix is unknown [1, 12]. Rosuvastatin dose adjustment may be considered based on clinical objectives or observations. Figure 9 summarizes the clinically relevant DDI results of elagolix effects on exposure of coadministered drugs, which informed dosing instructions in the USPI for Orilissa [1].

\subsection{DDIs with Oral Contraceptives}

As elagolix is prescribed to women of reproductive age and is not a contraceptive, it was important to evaluate the coadministration of elagolix and oral contraceptives (OCs). In a DDI study of elagolix and progestin-only contraception of norethindrone $0.35 \mathrm{mg}$ (i.e. mini-pill), the mean $C_{\max }$ and $\mathrm{AUC}_{24}$ values of norethindrone were comparable with and without coadministration of elagolix ( $\leq 12 \%$ change) [1], thus no dose adjustment was needed.

When elagolix $150 \mathrm{mg}$ once daily was administered with a triphasic OC containing doses of ethinylestradiol and norgestimate equivalent to ethinylestradiol $0.035 \mathrm{mg}$ and triphasic norgestimate $0.18 / 0.215 / 0.25 \mathrm{mg}$, ethinylestradiol $C_{\max }$ and $\mathrm{AUC}_{24}$ increased to approximately 1.15- and 1.30 -fold, respectively. There was a minimal decrease (up to $15 \%$ ) in exposures for the norgestimate metabolites norelgestromin and norgestrel [1]. These results indicate minimal PK drug interaction between elagolix and ethinylestradiol containing OCs. In addition, the combination of elagolix and norethindrone or elagolix and triphasic OCs did not negatively impact the hormonal effects of either elagolix or the $\mathrm{OC}$, therefore dose adjustment is not required. Based on the potential for E2-containing OCs to reduce the efficacy of elagolix, non-hormonal contraception is recommended.

\subsection{DDIs with Commonly Coadministered Drugs}

Two open-label DDI studies were conducted in healthy premenopausal females to evaluate the PK of elagolix alone and in combination with sertraline or fluconazole. Multipledose administration of sertraline ( $25 \mathrm{mg}$ once daily) did not affect the $300 \mathrm{mg}$ single-dose exposures $\left(C_{\max }\right.$ and AUC) of elagolix. Although, elagolix $300 \mathrm{mg}$ twice daily increased sertraline steady-state $C_{\max }$ and $\mathrm{AUC}_{24}$ to 1.34- and 1.42fold, respectively [1], such increases in sertraline exposures 


\section{Clinical Relevance}

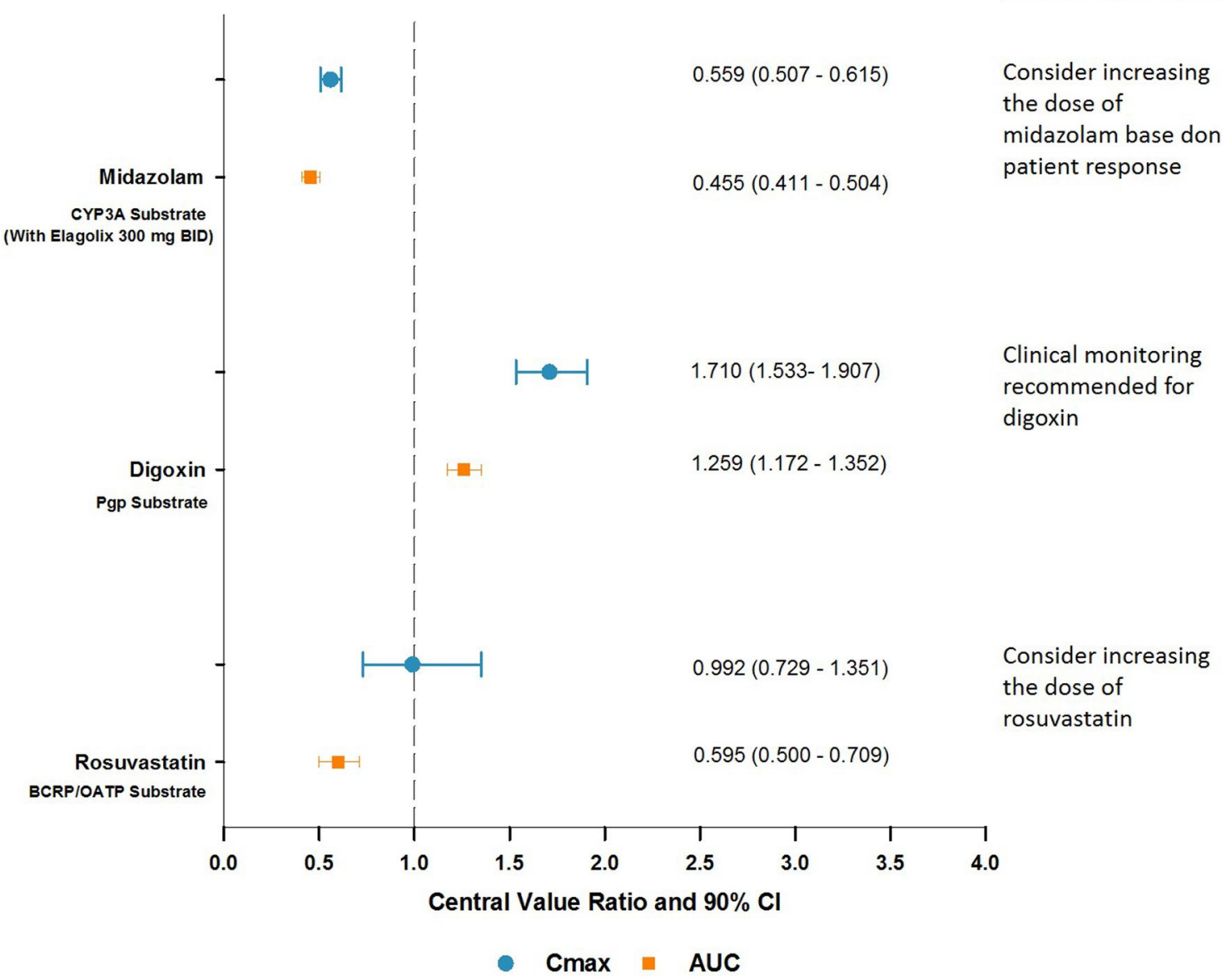

Fig. 9 Effect of elagolix on exposure of coadministered drugs, and clinical implications for dosing

are not considered clinically relevant given the wide safety margin of sertraline [19].

The steady-state exposures of elagolix $300 \mathrm{mg}$ twice daily $\left(C_{\max }\right.$ and $\mathrm{AUC}_{12}$ after 10 days) increased to 1.30 -fold following coadministration with a single dose of fluconazole $200 \mathrm{mg}$, whereas the mean exposures of fluconazole were unaltered compared with and without coadministration of elagolix [1].

Based on these results, dose adjustments are not required when elagolix is coadministered with sertraline or fluconazole.

\section{Population PK Analyses}

Population PK analyses were conducted using data from five phase I and four phase III studies in a total of 1624 subjects [8]. Elagolix population PKs were best described by a two-compartment model with a lag-time in absorption. The population mean $\mathrm{CL} / \mathrm{F}$ and $V_{\mathrm{c}} / \mathrm{F}$ were $118 \mathrm{~L} / \mathrm{h}$ and $257 \mathrm{~L}$, respectively. The interindividual variability on elagolix $\mathrm{CL} / \mathrm{F}$ and $V_{\mathrm{c}} / \mathrm{F}$ was $42.5 \%$ and $51 \%$, respectively.

OATP1B1 genotype status was the only significant covariate on elagolix PK parameters (i.e. specifically for CL/F). When subjects with a transporter genotype status of PT/IT were compared with ET/not genotyped, CL/F was reduced by only $14 \%$ [8], and when stratified by subjects with a transporter genotype status of PT or IT, elagolix CL/F was $44 \%$ and $20 \%$ lower, respectively, compared with subjects with a transporter genotype status of ET/not genotyped. The predicted increase in elagolix average concentrations $\left(C_{\text {avg }}\right)$ in subjects with OATP1B1 IT or PT relative to ET genotypes was $25 \%$ and $79 \%$, respectively. Despite these predicted increases in elagolix exposures in IT and PT subjects, the exposures were significantly overlapping with ET subjects, suggesting that these changes were not clinically meaningful. Thus, dose adjustment is not required for elagolix based on OATP1B1 genotype status.

None of the other tested covariates (age, body weight, race/ethnicity, hepatic or renal function) were significantly associated with elagolix PK parameters. In addition, PK 
exposures were similar between healthy women and women with endometriosis.

\section{Physiologically Based PK Analyses}

A PBPK model of elagolix was developed and verified using a combined bottom-up and top-down approach based on data from in vitro and several phase I single and multiple ascending dose, DDI, and special population studies [20]. The disposition pathways of elagolix, including metabolism by CYP3A and transport by P-gp, as well as hepatic uptake via OATP1B1, and their interplay, were quantified and verified using clinical DDI studies with prototypical inhibitors (rifampin and ketoconazole) and inducer (rifampin). A PBPK model of elagolix as a perpetrator was also verified using clinical DDI studies with a prototypical CYP3A substrate (midazolam) and a P-gp substrate (digoxin). The robust verification of this PBPK model provided confidence in predicting the DDI potential of elagolix as a perpetrator at the proposed clinical doses that were not evaluated in clinical DDI studies.

Based on the PBPK model prediction, elagolix could be classified as a moderate inducer of CYP3A at a $200 \mathrm{mg}$ twice-daily dose (approximately 56\% reduction in midazolam AUC). Elagolix $150 \mathrm{mg}$ once daily is predicted to increase digoxin AUC by less than 1.25-fold (approximately $19 \%$ increase in digoxin AUC); however, since elagolix is predicted to increase the $C_{\max }$ of digoxin by $68 \%$ following a single $150 \mathrm{mg}$ dose, the simulation results indicated that monitoring patients receiving digoxin along with elagolix (150 mg once daily or $200 \mathrm{mg}$ twice daily) is recommended [1].

Overall, the PBPK model represents a novel approach, accounting for interplay between metabolism and transport. The model was applied to evaluate the potential DDI of elagolix under various dosages and clinical regimens as a victim with $\mathrm{P}$-gp, OATP, and CYP3A4 modulators, and as a perpetrator for CYP3A and P-gp substrates.

\section{Exposure-Response Relationship}

\subsection{Exposure-Efficacy Analysis for Dose Justification}

Data from four phase III studies in premenopausal women with moderate to severe pain associated with endometriosis were included in a population PK analysis to describe the relationship between elagolix exposure and the clinical efficacy response variables of dysmenorrhea (DYS; pain with menstruation) and non-menstrual pelvic pain
(NMPP). A discrete-time, first-order Markov chain model adequately described the relationship between elagolix monthly $C_{\text {avg }}$ values and transition probabilities between responder and non-responder states. Additionally, the model included transitions to account for the subject dropouts from responder state, non-responder state, and after month 6 , and adequately predicted subject dropouts over time with placebo and elagolix treatments. The elagolix monthly $C_{\text {avg }}$ values calculated over the preceding month were a better predictor of DYS and NMPP responses than trough concentration $\left(C_{\text {trough }}\right)$ values [21].

Demographics (body size measurements, race, ethnicity, and geographic region), baseline hormone levels, disease severity measures (number of days and intensity of bleeding), time since endometriosis diagnosis, baseline analgesic use, alcohol or tobacco use, and prior GnRH therapies were not statistically significant covariates in the model, indicating that these covariates do not influence DYS and NMPP responder rates. On the other hand, baseline DYS and NMPP scores were significant covariates on respective placebo transition probabilities, with higher placebo response in subjects with higher baseline disease scores. The final model demonstrated strong exposure-efficacy relationships for both primary endpoints (DYS and NMPP), adequately described the phase III efficacy data for both elagolix dose regimens (150 mg once daily and $200 \mathrm{mg}$ twice daily), and provided supportive evidence for the approval of Orilissa endometriosis doses [21].

\subsection{Exposure-Safety Analysis to Support the Duration of Treatment}

Exposure-bone mineral density (BMD) modeling using data from the four phase III studies noted above revealed an exposure-response relationship between elagolix $C_{\text {avg }}$ and changes in BMD. An indirect response model was developed to describe the effect of elagolix $C_{\text {avg }}$ on BMD and to evaluate the significance of clinically relevant covariates. The exposure-BMD model results showed that the estimated half maximal effective concentration $\left(\mathrm{EC}_{50}\right)$ of elagolix for reduction in BMD was $240 \mathrm{ng} / \mathrm{mL}$, a concentration well above (more than fivefold) the plasma exposure associated with $150 \mathrm{mg}$ once-daily dosing $\left(C_{\text {avg }}\right.$ concentrations of approximately $47 \mathrm{ng} / \mathrm{mL}$ ). This result is consistent with the small BMD change with $150 \mathrm{mg}$ once-daily dosing (approximately - 1\% BMD change from baseline after 12 months) [21].

Final model results showed that African American race, higher baseline body mass index (BMI) and lower C-terminal telopeptide (CTX) levels (a bone resorption biomarker) were significant predictors of higher baseline BMD. African American race has been previously shown to be associated with higher BMD compared with other race groups in the 
Table 2 Summary statistics of predicted percentage change from baseline in lumbar spine bone mineral density following treatment with elagolix $150 \mathrm{mg}$ qd or $200 \mathrm{mg}$ bid for 36 months

\begin{tabular}{lllll}
\hline Month & \multicolumn{4}{l}{ Percentage change from baseline in lumbar spine BMD [mean (95\% CI)] } \\
\cline { 2 - 4 } & $150 \mathrm{mg} \mathrm{qd}$ & & \multicolumn{2}{l}{$200 \mathrm{mg}$ bid } \\
\cline { 2 - 4 } & Model & Observed $^{\mathrm{a}}$ & Model & Observed $^{\mathrm{a}}$ \\
\hline 6 & $-0.6(-1.1$ to -0.2$)$ & $-0.3,-0.7$ & $-1.6(-2.0$ to -1.2$)$ & $-2.6,-2.5$ \\
12 & $-1.1(-1.5$ to -0.5$)$ & $-0.6,-1.1$ & $-2.7(-3.3$ to -2.2$)$ & $-3.6,-3.9$ \\
24 & $-1.6(-2.3$ to -0.9$)$ & NA & $-4.3(-5.2$ to -3.7$)$ & NA \\
36 & $-1.9(-2.9$ to -1.2$)$ & NA & $-5.2(-6.2$ to -4.3$)$ & NA \\
\hline
\end{tabular}

bid twice daily, $B M D$ bone mineral density, $C I$ confidence interval, $N A$ not available, $q d$ once daily

${ }^{a}$ Mean from each phase III study [4, 27]
US [22, 23]. Similarly, BMI, body fat, and body weight have all been shown to be associated with higher BMD [24-26]. In addition to its effects on baseline BMD, BMI was also significantly associated with higher bone formation rates $\left(K_{\text {in }}\right)$. After incorporating the above covariates, none of the tested covariates (including baseline BMD, expressed as a $Z$ score) were significantly associated with BMD changes due to elagolix treatment [21].

The final exposure-BMD model was used to simulate a longer duration of treatment beyond the phase III, placebocontrolled, 6-month and extension studies (up to 12 months continuous treatment). The model predicted BMD changes at months 6 and 12 were consistent with the observed data in the pivotal and extension phase III trials (Table 2). Simulations of 36 months of continuous treatment of elagolix $150 \mathrm{mg}$ once daily or $200 \mathrm{mg}$ twice daily suggested that the mean percentage change from baseline in lumbar spine BMD is approximately $-2 \%$ and $-5 \%$ for each regimen, respectively (Table 1) [12]. These analyses supported approval of the indicated use of Orilissa $150 \mathrm{mg}$ once daily or $200 \mathrm{mg}$ twice daily for 24 months, or 6 months of continuous duration of treatment, respectively [1].

\section{Conclusions}

Elagolix clinical pharmacology characteristics, underlying sources of population variability, and the exposure-response (safety and efficacy) relationships were extensively characterized in clinical studies in healthy subjects and in women with endometriosis. Elagolix clinical pharmacology attributes consist of linear PK over the efficacious dose range (150 mg once daily to $200 \mathrm{mg}$ twice daily), and rapid PD onset and offset, leading to dose-dependent partial suppression (150 mg once daily) and near full suppression (200 mg twice daily) of gonadotropin and ovarian hormones. Elagolix is eliminated primarily via hepatic metabolism by CYP3A, its bioavailability is not significantly impacted by food, has a manageable drug-drug interaction profile with most coadministered medications (only one contraindication with strong OATP1B1 inhibitors), and is contraindicated in women with severe hepatic impairment (Child-Pugh C). Elagolix is a weak to moderate inducer of CYP3A and an inhibitor of P-gp, as demonstrated by the decreased exposure of midazolam (increasing the dose of midazolam may be considered) and increased exposure of digoxin (clinical monitoring is recommended) when coadministered with elagolix. Elagolix does not prolong the QTc interval at supratherapeutic doses and exposures. Elagolix exposures are not affected by any degree of renal impairment or mild hepatic (Child-Pugh A) impairment, while threeand sevenfold increases in elagolix exposure were observed in Child-Pugh B and C subjects, respectively. The phase III population PK analysis demonstrated a minimal impact of patients' characteristics and demographics on the PK of elagolix. It is worth highlighting that with elagolix $200 \mathrm{mg}$ twice daily, E2 suppression is near maximal, and further increases in elagolix exposures due to intrinsic or extrinsic factors may not lead to further significant changes in E2 levels and the downstream hypoestrogenic effects. Elagolix demonstrated an exposure-response relationship with hormonal changes, as well as primary efficacy (DYS and NMPP) and safety (BMD changes) endpoints. Since the BMD changes observed with elagolix were time-dependent $[4,27]$, the exposure-BMD model enabled simulations of various durations of treatment that ultimately supported the 24 months' approved duration of use with $150 \mathrm{mg}$ once daily with no coexisting conditions (dyspareunia or Child-Pugh B). Overall, the full characterization of the elagolix clinical pharmacology profile and the model-based analyses played a critical role in the approval of elagolix as the first oral GnRH receptor antagonist for the management of moderate to severe pain associated with endometriosis [1].

Acknowledgements The authors thank Ms. Denise Beck, Dr. Sven Stodtmann, and Dr. Ahmed Suleiman for their help in the modeling analyses; Dr. Michael Snabes and Dr. Keith Gordon for their help in developing the mechanism of action figure; and Dr. Pooja Manchandani for developing the estradiol levels figure. The authors also would like to thank Ms. Martha Ewen for her help in writing reports and regulatory documents, and Neurocrine for conducting the human 
absorption, distribution, metabolism, and excretion studies, and the regional absorption studies.

\section{Compliance with Ethical Standards}

Funding This work was supported by AbbVie, who contributed to the study design, research, and interpretation of the data, and the writing, review, and approval of the manuscript.

Conflict of interest All authors are employees of AbbVie and may hold AbbVie stock or stock options. Medical writing support was provided by Therese Stickler, a freelance writer under contract with AbbVie.

Open Access This article is distributed under the terms of the Creative Commons Attribution-NonCommercial 4.0 International License (http://creativecommons.org/licenses/by-nc/4.0/), which permits any noncommercial use, distribution, and reproduction in any medium, provided you give appropriate credit to the original author(s) and the source, provide a link to the Creative Commons license, and indicate if changes were made.

\section{References}

1. Orilissa ${ }^{\mathrm{TM}}$ (elagolix) [United States package insert]. North Chicago, IL. AbbVie Inc. 2018.

2. Archer DF, Stewart EA, Jain RI, Feldman RA, Lukes AS, North $\mathrm{JD}$, et al. Elagolix for the management of heavy menstrual bleeding associated with uterine fibroids: results from a phase 2a proofof-concept study. Fertil Steril. 2017;108(1):152-60.e4

3. Diamond M, Soliman AM, Gao J, Owens C, Chwalisz K, Archer DF. Elagolix improves quality of life in women with heavy menstrual bleeding associated with uterine fibroids: evidence from a phase 2b randomized trial. Fertil Steril. 2017;108(3):e27.

4. Taylor HS, Giudice LC, Lessey BA, Abrao MS, Kotarski J, Archer DF, et al. Treatment of endometriosis-associated pain with elagolix, an oral GnRH antagonist. N Engl J Med. 2017;377(1):28-40.

5. Ng J, Chwalisz K, Carter DC, Klein CE. Dose-dependent suppression of gonadotropins and ovarian hormones by elagolix in healthy premenopausal women. J Clin Endocrinol Metab. 2017;102(5):1683-91.

6. Dun EC, Taylor HS. Elagolix: a promising oral GnRH antagonist for endometriosis-associated pain. Oncotarget. 2017;8(59):99219-20

7. Chen C, Wu D, Guo Z, Xie Q, Reinhart GJ, Madan A, et al. Discovery of sodium R-(+)-4-\{2-[5-(2-fluoro-3-methoxyphenyl)3-(2-fluoro-6-[trifluoromethyl]benzyl)-4 -methyl-2,6-dioxo3,6-dihydro-2H-pyrimidin-1-yl]-1-phenylethylamino \}butyrate (elagolix), a potent and orally available nonpeptide antagonist of the human gonadotropin-releasing hormone receptor. J Med Chem. 2008;51(23):7478-85.

8. Winzenborg I, Nader A, Polepally AR, Liu M, Degner J, Klein $\mathrm{CE}$, et al. Population pharmacokinetics of elagolix in healthy women and women with endometriosis. Clin Pharmacokinet. 2018;57(10):1295-306.

9. Ng J, Salem A, Carter D, Williams LA, Klein CE. Effect of the coadministration of ketoconazole on the pharmacokinetics and safety of elagolix in healthy premenopausal females. Clin Pharmacol Ther. 2016;99:S55

10. Struthers RS, Nicholls AJ, Grundy J, Chen T, Jimenez R, Yen SS, et al. Suppression of gonadotropins and estradiol in premenopausal women by oral administration of the nonpeptide gonadotropin-releasing hormone antagonist elagolix. J Clin Endocrinol Metab. 2009;94(2):545-51.

11. Archer D, Ng J, Chiu Y, Klein C, Chwalisz K. Dose dependent suppression of ovulation and ovarian activity by elagolix in healthy premenopausal women. Reproductive Sciences. 2017;24(Suppl 1):140A.

12. Elagolix Multidiscipline Review, US FDA Center for Drug Evaluation and Research. 2018. https://www.accessdata.fda.gov/drugs atfda_docs/nda/2018/210450Orig1s000MultiD.pdf. Accessed July 2018 .

13. $\mathrm{Ng}$ J, Klein CE, Awni WA, Williams LA. Pharmacokinetics of elagolix, an oral gonadotropin-releasing hormone $(\mathrm{GnRH})$ antagonist, in healthy premenopausal Han Chinese and Japanese female subjects. In: Presented at the 12th World Congress on Endometriosis. 30 April-3 May 2014: Sao Paulo.

14. Ng J, Duan WR, Marbury T, Schmidt JM, Klein CE. Elagolix pharmacokinetic profiles in women with renal or hepatic impairment. Clin Pharmacol Drug Dev. 2019;8(8):1053-61.

15. Clinical Drug Interaction Studies-Study Design, Data Analysis, and Clinical Implications. US Department of Health and Human Services Food and Drug Administration Center for Drug Evaluation and Research (CDER). 2017.

16. Ng J, Salem A, Carter D, Williams LA, Klein CE. Effects of the coadministration of single and multiple doses of rifampin on the pharmacokinetics and safety of elagolix in healthy premenopausal females. Clin Pharmacol Ther. 2016;99:S54-5.

17. Polepally AR, Dufek MB, Dharia SP, Kamradt K, Lin J, Peloso $\mathrm{PM}$, et al. Drug-drug interaction of midazolam with elagolix, a novel oral GnRH antagonist: analyses of data from two studies. Clin Pharmacol Drug Dev. 2017;6:48.

18. Ng J, Salem A, Carter D, Klein CE. Effects of the coadministration of multiple doses of elagolix on the pharmacokinetics and safety of digoxin in healthy women. Clin Pharmacol Drug Dev. 2017;6:54-5.

19. Cooper JM, Duffull SB, Saiao AS, Isbister GK. The pharmacokinetics of sertraline in overdose and the effect of activated charcoal. Br J Clin Pharmacol. 2015;79(2):307-15.

20. Chiney MS, Ng J, Gibbs JP, Shebley M. Quantitative assessment of elagolix enzyme-transporter interplay and drug-drug interaction using physiologically-based pharmacokinetic modeling. Clin Pharmacokinet. 2019. https://doi.org/10.1007/s40262-019-00833 -6. (in press)

21. Nader A, Winzenborg I, Suleiman A, Polepally A, Ng J, Shebley $\mathrm{M}$, et al. Combined efficacy and safety modeling and simulation approach to inform drug development decisions for an oral gonadotropin-releasing hormone $(\mathrm{GnRH})$ receptor antagonist. J Pharmacokinet Pharmacodyn. 2018;45(Suppl 1):S4.

22. Nam HS, Kweon SS, Choi JS, Zmuda JM, Leung PC, Lui LY, et al. Racial/ethnic differences in bone mineral density among older women. J Bone Miner Metab. 2013;31(2):190-8.

23. Wilkin LD, Jackson MC, Sims TD, Haddock BL. Racial/ethnic differences in bone mineral density of young adults. Int J Exerc Sci. 2010;3(4):197-205.

24. Reid I. Fat and bone. Arch Biochem Biophys. 2010;503(1):20-7.

25. Lidell ME, Enerback S. Brown adipose tissue and bone. Int J Obes Suppl. 2015;5(Suppl 1):S23-7.

26. Norgan NG. The beneficial effects of body fat and adipose tissue in humans. Int J Obes Relat Metab Disord. 1997;21(9):738-46.

27. Surrey E, Taylor HS, Giudice L, Lessey BA, Abrao MS, et al. Long-term outcomes of elagolix in women with endometriosis: results from two extension studies. Obstet Gynecol. 2018;132(1):147-60.

28. Carr BR, Stewart EA, Archer DF, Al-Hendy A, Bradley L, et al. Elagolix alone or with add-back therapy in women with heavy menstrual bleeding and uterine leiomyomas: a randomized controlled trial. Obstet Gynecol. 2018;132(5):1252-64. 